\title{
Screening of a natural compound library identifies emodin, a natural compound from Rheum palmatum Linn that inhibits DPP4
}

\author{
Zhaokai Wang ${ }^{1,2}$, Longhe Yang ${ }^{3}$, Hu Fan ${ }^{2}$, Peng Wu ${ }^{2}$, Fang Zhang ${ }^{2}$, Chao Zhang ${ }^{4}$, Wenjie Liu ${ }^{\text {Corresp., }}{ }^{5}$, \\ Min Li Corresp. 1 \\ ${ }^{1}$ College of Life Sciences, Fujian Normal University, Fuzhou, P. R. China \\ 2 Engineering Research Center of Marine Biological Resource Comprehensive Utilization, Third Institute of Oceanography, State Oceanic Administration, \\ Xiamen, P. R. China \\ 3 Engineering Research Center of Marine Biological Resource Comprehensive Utilization, Third Institute of Oceanography, State Oceanic Administration, \\ Subject Areas, Xiamen, P. R. China \\ 4 Engineering Research Center of Marine Biological Resource Comprehensive Utilization, Third Institute of 10 Oceanography, State Oceanic \\ Administration, Xiamen, P. R. China \\ 5 Fujian Provincial Key Laboratory of Innovative Drug Target Research, School of Pharmaceutical Sciences, Xiamen, P. R. China \\ Corresponding Authors: Wenjie Liu, Min Li \\ Email address: wjliu@xmu.edu.cn, mli@fjnu.edu.cn
}

Historically, Chinese herbal medicines have been widely used in the treatment of hyperglycemia, but the mechanisms underlying their effectiveness remain largely unknown. Here, we screened a compound library primarily comprising natural compounds extracted from herbs and marine organisms. The results showed that emodin, a natural compound from Rheum palmatum Linn, inhibited DPP4 activity with an in vitro IC $\mathrm{IC}_{50}$ of 5.76 $\mu \mathrm{M}$ without inhibiting either DPP8 or DPP9. A docking model revealed that emodin binds to DPP4 protein through Glu205 and Glu206, although with low affinity. Moreover, emodin treatment (3, 10 and $30 \mathrm{mg} / \mathrm{kg}$, P.O.) in mice decreased plasma DPP4 activity in a dosedependent manner. Our study suggests that emodin inhibits DPP4 activity and may represent a novel therapeutic for the treatment of type 2 diabetes. 


\section{Screening of a natural compound library identifies \\ 3 emodin, a natural compound from Rheum palmatum \\ 4 Linn that inhibits DPP4}

5 Zhaokai Wang ${ }^{1,2}$, Longhe Yang ${ }^{2}$, Fan $\mathrm{Hu}^{2}$, Peng $\mathrm{Wu}^{2}$, Fang $\mathrm{Zhang}^{2}$, Chao Zhang ${ }^{2}$,Wenjie Liu ${ }^{3 *}$ and Min

$6 \mathbf{L i}^{*}$

\section{Author and article information}

$8{ }^{1}$ College of Life Sciences, Fujian Normal University. Fuzhou, 350007, China;

$9 \quad{ }^{2}$ Engineering Research Center of Marine Biological Resource Comprehensive Utilization, Third Institute of Oceanography, State

10 Oceanic Administration, Xiamen, Fujian 361005, P. R. China;

$11{ }^{3}$ Fujian Provincial Key Laboratory of Innovative Drug Target Research, School of Pharmaceutical Sciences, Xiamen University,

12 Xiamen, Fujian, 361102, P. R. China

13 E-Mails: wang@tio.org.cn (Z.W.); longheyang@tio.org.cn (L.Y.); fhu@tio.org.cn (F.H.); pwu@tio.org.cn (P.W.); fzhang@tio.org.cn (F.Z); czhang@tio.org.cn (C.Z.).

$15 *$ Corresponding author:

wjliu@xmu.edu.cn(W.L.),Tel.: +86-592-2881180; mli@fjnu.edu.cn(M.L.), Tel.+86-591-22867555.

\section{Abstract}

Historically, Chinese herbal medicines have been widely used in the treatment of hyperglycemia, but the mechanisms underlying their effectiveness remain largely unknown. Here, we screened a compound library primarily comprising natural compounds extracted from herbs and marine organisms. The results showed that emodin, a natural compound from Rheum palmatum Linn, inhibited DPP4 activity with an in vitro $\mathrm{IC}_{50}$ of $5.76 \mu \mathrm{M}$ without inhibiting either DPP8 or DPP9. A docking model revealed that emodin binds to DPP4 protein through Glu205 and Glu206, although with low affinity. Moreover, emodin treatment (3,10 and $30 \mathrm{mg} / \mathrm{kg}$, P.O.) in mice decreased plasma DPP4 activity in a dosedependent manner. Our study suggests that emodin inhibits DPP4 activity and may represent a novel therapeutic for the treatment of type 2 diabetes.

\section{Main article text}

\section{Introduction}

Type 2 diabetes mellitus (T2DM) is a metabolic disease associated with insulin resistance and pancreatic $\beta$-cell failure (Defronzo 2009). Therefore, enhancing pancreatic insulin secretion while protecting pancreatic $\beta$-cells represents a promising therapeutic approach for the treatment of type 2 diabetes. Glucagon-like peptide 1 (GLP-1) is one of the incretin hormones released from cells in the gastrointestinal tract in response to nutrient absorption. Incretin hormones, especially GLP-1, regulate post-prandial insulin secretion by inhibiting glucagon release and stimulating insulin biosynthesis and secretion (Baggio \& Drucker 2007). In T2DM patients, GLP-1 is critical for glucose homeostasis (Mulvihill \& Drucker 2014).

Dipeptidyl peptidase 4 (DPP4), which was first identified by Hopsu-Havuand Glenner, rapidly 
degrades the active form of GLP-1 (GLP- $1_{7-36}$ ) to inactive GLP-19-36 within minutes in vivo (Hopsu-Havu \& Glenner 1966; Mulvihill \& Drucker 2014). DPP4 is commonly expressed as two forms: a membraneassociated and soluble circulating protein and a cleaved protein containing either analanine or proline at position 2 (Lambeir et al. 2003). Therefore, a DPP4 inhibitor could potentially increase the effect of intact GLP-1, thus prolonging its anti-diabetic effects (Smith et al. 2014).

Although several DPP4 inhibitors such as sitagliptin (MK-0431) (Kim et al. 2005), vildagliptin (LAF237) (Villhauer et al. 2003), saxagliptin (BMS-477118) (Augeri et al. 2005), alogliptin (SYR-322) (Feng et al. 2007) and linagliptin (BI-1356) (Eckhardt et al. 2007) have been approved for the treatment of T2DM, few natural compounds have been reported to exert DPP4 inhibitory activity (Geng et al. 2013).

Traditional Chinese medicine (TCM) has been used in the clinical treatment of diabetes and related complications for centuries (Wang \& Chiang 2012; Xie \& Du 2011). Radix Astragali (Wang et al. 2009) and Radix Rehmanniae (Huang et al. 2010) are TCMs with both hypoglycemic and anti-inflammatory activities as reviewed by Xie et al. (Xie \& Du 2011) and Liu et al.(Liu et al. 2004). However, the underlying mechanisms of the effective components are largely unknown because of the poor characterization of Chinese medicine. Herein, we screened a small library of natural products from Chinese herbal medicines and marine organisms to identify new molecules that inhibit DPP4 activity. In our research, we discovered that emodin from the herb Rheum palmatum Linn inhibited DPP4 activity with an $\mathrm{IC}_{50}$ of 5.76 $\mu \mathrm{M}$ without inhibiting of either DPP8 or DPP9. Moreover, oral administration of emodin decreased DPP4 activity in a dose-dependent manner in mice.

\section{Materials and Methods}

\section{Materials}

The natural product library derived from Chinese herbs was purchased from Selleck Chemicals (Cat\# L1400, Shanghai, China). Marine-derived compounds were isolated and purified from marine organisms in our lab.

\section{DPP4 activity assay}

The DPP4 screening assay was conducted using a DPP4 inhibitor screening assay kit (Cayman Chemical, Ann Arbor, MI), following the manufacturer's protocol. Briefly, $30 \mu \mathrm{l}$ of diluted assay buffer, $10 \mu \mathrm{l}$ of diluted DPP4, and $10 \mu \mathrm{l}$ of inhibitor were added to a 96-well plate. The reaction was initiated by adding $50 \mu \mathrm{l}$ of diluted substrate solution to all of the wells, and this was followed by incubation with a plate cover at $37^{\circ} \mathrm{C}$ for 30 minutes. After incubation, the fluorescence was read using an excitation wavelength of $360 \mathrm{~nm}$ and an emission wavelength of $460 \mathrm{~nm}$.

\section{DPP8 activity assay}

A DPP8 assay kit was purchased from BPS Bioscience (Cat\# 800208), and the assay protocol was followed to test for inhibitory activity on DPP8. Briefly, DPP substrate 1 was diluted to make a $100 \mu \mathrm{M}$ stock solution, and DPP8 protein was diluted in DPP assay buffer to $2 \mathrm{ng} / \mu \mathrm{l}(20 \mathrm{ng} /$ reaction). For the tested compounds, $10 \mu \mathrm{l}$ of diluted DPP8 protein, $5 \mu$ l of diluted DPP substrate $1,84 \mu \mathrm{l}$ of DPP assay buffer and $1 \mu \mathrm{l}$ of inhibitor were added into the assay system for a total volume of $100 \mu$ l. The reaction mixtures were prepared in duplicate on a 96-well plate and incubated at room temperature for 10 minutes. The plate was read on an Envision plate reader (Perkin-Elmer) capable of excitation at $365 \mathrm{~nm}$ and emission detection at $460 \mathrm{~nm}$. 
79 A DPP9 assay kit was purchased from BPS Bioscience (Cat\# 800209), and the assay protocol was followed 80 to test for inhibitory activity against DPP9. Briefly, DPP substrate 1 was diluted to make a $100 \mu \mathrm{M}$ stock 81 solution, and DPP9 protein was diluted in DPP assay buffer to $2 \mathrm{ng} / \mu \mathrm{l}$ ( $20 \mathrm{ng} /$ reaction). For the tested 82 compounds, $10 \mu \mathrm{l}$ of diluted DPP9 protein, $5 \mu \mathrm{l}$ of diluted DPP substrate 1, $84 \mu \mathrm{l}$ of DPP assay buffer and $831 \mu \mathrm{l}$ of inhibitor were added into the assay system for a total volume of $100 \mu \mathrm{l}$. The reaction mixtures 84 were prepared in duplicate on a 96-well plate and incubated at room temperature for 10 minutes. The 85 plate was read on an Envision plate reader (Perkin-Elmer) capable of excitation at $365 \mathrm{~nm}$ and emission 86 detection at $460 \mathrm{~nm}$.

\section{Docking assay}

88 Docking of compounds to the DPP4 active site was modeled using the Glide package. The 3-dimensional model of DPP4 (PDB code: 2ONC) was used in the molecular modeling experiment (Huang et al. 2010). Compounds were docked onto the DPP4 binding site at a position in which either the substrate or small molecule inhibitors were fitted into the active pocket. Bond formation between the compound and the DPP4 active site was dynamically simulated.

\section{Dialysis assay}

Dialysis assay was performed using Slide-A-Lyzer Dialysis Cassettes (Pierce, Shanghai, China). Briefly, 2 mg DPP4 protein was incubated with emodin or dimethyl sulfoxide (DMSO) in $4 \mathrm{ml}$ diluted assay buffer for 10 minutes at $37^{\circ} \mathrm{C}$. Mixed reaction solution was loaded onto a dialysis cartridge using a syringe and incubated at $4^{\circ} \mathrm{C}$ for $8 \mathrm{~h}$. The samples were removed from Dialysis Cassettes by syringes for DPP4 assay.

\section{Animal study}

99 Balb/c mice (male, 6 weeks) and ob/ob (-/-) mice (male, 6 weeks) were purchased from the Shanghai SLAC Laboratory Animal Co. Ltd. (Shanghai, China) and maintained in an air-conditioned room at 20$25^{\circ} \mathrm{C}$ under a $12 \mathrm{~h}$ dark/light cycle and fed certified standard chow and tap water ad libitum. Experiments were conducted in compliance with the Guide for the Care and Use of Laboratory Animals. Mice were orally administered with emodin $(3,10$, or $30 \mathrm{mg} / \mathrm{kg}$ ) and had their blood collected at $0.5,1,2$, and 4 hours after emodin treatment. The samples were subjected to plasma isolation immediately after collection. Plasma samples were tested for DPP4 activity with a DPP4-Glo assay kit (Promega, Beijing,

106 China) according to the manufacturer's protocol. The experimental protocol was approved by Animal Care and Use Committee of Xiamen University(XM2015030514).

\section{Data analysis}

109 Results are presented as the mean \pm standard error (SEM). Differences between the groups were analyzed

110 using multiple variances (one-way ANOVA or two-way ANOVA) followed by Bonferroni's test, with 111 GraphPad Prism 5 software (GraphPad Software, San Diego, CA, USA). Differences were considered to 112 be statistically significant at $\mathrm{p}<0.05$.

\section{Results and Discussion}

\section{Emodin inhibits DPP4 activity in vitro}


115 To screen for novel DPP4 inhibitors from natural compounds, we established a natural compound library

116 comprising 155 naturally derived compounds, in which 131 were isolated and purified from Chinese

117 herbal medicines, and 24 were from marine organisms. DPP4 screening was first conducted on these 155

118 natural compounds by following a DPP4 screening assay kit protocol. All compounds $(10 \mu \mathrm{M})$ were

119 screened for DPP4 inhibitory activity. The results suggested that emodin showed greater than 50\%

120 inhibition in the DPP4 activity assay at $10 \mu \mathrm{M}$. Two other compounds were ruled out because of auto-

121 fluorescence (Figure 1A).

122 To further validate this finding, a dose response experiment was performed to test the inhibitory

123 activity of emodin on DPP4. Emodin was shown to inhibit DPP4 activity in vitro with an $\mathrm{IC}_{50}$ of $5.76 \mu \mathrm{M}$

124 and Ki of 0.85 (Figure 1B). The DPP4 antagonist sitagliptin was used as a positive control, which showed

125 an $\mathrm{IC}_{50}$ of $21.78 \mathrm{nM}$ (Figure 1C), a value similar to those from previous reports (Kim et al. 2005).

126 Considering that emodin is an anthraquinone, and many naturally occurring anthraquinones have

127 been identified as having anti-diabetes activity (Chen et al. 2015; Lin et al. 2015; Ramos-Zavala et al. 2011;

128 Wu et al. 2014), we wondered whether this class of compounds, such as Aloe-emodin, rheochrysidin,

129 chrysophanol and rhein, might inhibit DPP4 activity.

130 Anthraquinone compounds inhibit DPP4 activity but not DPP8 or DPP9 activity

131 We further investigated a series of anthraquinone compounds in the DPP4 activity assay to identify 132 potent DPP4 inhibitors in this class. Aloe-emodin, rheochrysidin, chrysophanol and rhein were tested by 133 using the same DPP4 assay format (Table 1). The $\mathrm{IC}_{50}$ of each compound is listed, with aloe-emodin 134 showing an $\mathrm{IC}_{50}$ of $16.02 \mu \mathrm{M}$ and rhein showing an $\mathrm{IC}_{50}$ of $23.06 \mu \mathrm{M}$. The $\mathrm{IC}_{50}$ values of rheochrysidin and 135 chrysophanol were greater than $100 \mu \mathrm{M}$ (Table 1). These results showed that emodin was the most 136 effective anthraquinone in inhibiting DPP4 activity. In addition, the Ki and binding energy for these 137 compounds have also been listed in Table 1. Because of the high similarity between DPP4 and DPP8/9 138 and the reported toxicity of DPP8 or DPP9 inhibition in animal studies (Lankas et al. 2005), we tested the 139 anthraquinone compounds in DPP8 and DPP9 activity assays. All of the compounds were tested at 100 $140 \mu \mathrm{M}$, and none showed activity against either DPP8 or DPP9. Rhein showed a very weak activity on 141 DPP8, with an IC50 greater than $100 \mu \mathrm{M}$. The biological function of rhein on DPP8 is minimal compared 142 to other DPP8 inhibitors. This result suggests that emodin is a relatively selective inhibitor against DPP4 143 (Figure 2). To confirm this finding, we conducted a molecular docking assay.

Emodin binds to Glu205 and Glu206 of DPP4 protein in a docking model

145 The active site of DPP4 consists of Arg125, Glu205, Glu206, Typ547, Trp629, Tyr666, and His740 146 according to the crystal structure template of DPP4 with a small molecular inhibitor (PDB code: 2ONC) 147 (Feng et al. 2007). Our docking model revealed that the negatively charged hydroxyl group of emodin is 148 engaged in tight H-bonding with Glu205 and Glu206 (Fig. 3A), suggesting a mechanism of binding of 149 emodin to the DPP4 active site. The binding modes showed that emodin was bound to the active site of 150 DPP4 with the hydroxyl moiety but did not form hydrogen bonds with other amino moieties such as 151 Tyr547 or Trp629 (Ji et al. 2014; Kim et al. 2005), which may affect the activity of emodin. The 152 compounds with similar structure as emodin that have hydroxyl group at similar site could also form $\mathrm{H}$ 153 bond with Glu205 and Glu206, and these compounds (aloe-emodin and rhein) also showed DPP4 154 inhibitory activity. In comparison, compounds without the hydroxyl group at R2 location (rheochrysidin 155 and chrysophanol) lack the ability to form H-bond with DPP4 at active site, thus they showed weakest 156 DPP4 inhibitory activity.Following dialysis assay suggested emodin binding to the DPP4 active site in a 
157 reversible manner (Fig. 3B). To evaluate the DPP4 inhibitory activity of emodin in vivo, we orally 158 administered emodin to Balb/c mice.

\section{Emodin inhibits DPP4 activity in vivo}

160 DPP4, also known as adenosine deaminase complexing protein 2 or T-cell activation antigen CD26, is a member of the large family of proteases. DPP4 is associated with immune regulation, signal transduction and apoptosis. Recent reports shown that DPP4 correlates closely to diabetes and cancer. Our labs have focused attention on DPP4 for the screening of inhibitors, such as emodin from Rheum palmatum Linn. As a natural product and active ingredient of various Chinese herbs, emodin exerts its anti-diabetic effects partially by upregulating the expression of the pancreas L-type calcium channel in streptozotocin (STZ)-induced dyslipidemic diabetic rats (Zhao et al. 2009) and by inhibiting 11 beta-hydroxysteroid dehydrogenase type 1 (11 $\beta$-HSD1) activity in diet-induced obese (DIO) mice (Feng et al. 2010; Wang et al. 2012). Xue et al. have also reported that emodin exerts anti-diabetic effects against PPAR-gamma in mice either administered a high-fat diet or treated with low-dose STZ to induce diabetes (Xue et al. 2010). Song et al. have reported that emodin regulates glucose homeostasis in vivo via AMP-activated kinase (AMPK) activation (Song et al. 2013). Emodin has also been shown to decrease blood glucose in rats with diabetes induced by low-dose STZ combined with high energy intake (Wu et al. 2014). These data clearly show that emodin exerts anti-hypoglycemic effects through diverse mechanisms, which is in line with the results of our screening analysis.

To test the inhibitory activity of emodin on DPP4 in vivo, an animal experiment was conducted by oral administration of emodin $(3,10$ and $30 \mathrm{mg} / \mathrm{kg}$, P.O., $\mathrm{n}=5)$ to Balb/c mice, followed by plasma collection at different time points to measure DPP4 activity in the blood. Plasma samples were collected at 0, 0.5, 1, 2 and 4 hours after the oral dose of emodin, and plasma DPP4 activity was measured with a DPP4-Glo assay kit. The results suggested that emodin treatment $(3,10$ and $30 \mathrm{mg} / \mathrm{kg}$, P.O.) in mice decreased the plasma DPP4 activity in a dose-dependent manner (Fig. 4). The lowest does of emodin ( $3 \mathrm{mg} / \mathrm{kg})$ decreased plasma DPP4 activity from baseline, although these levels rebounded after 1 hour, whereas 10 and $30 \mathrm{mg} / \mathrm{kg}$ doses of emodin decreased plasma DPP4 activity and maintained the lower levels until 2 hours post-treatment (Fig. 4). This dose-dependent manner is probably due to the pharmacokinetics of emodin, and 10 and $30 \mathrm{mg} / \mathrm{kg}$ have maximized the pharmacokinetic coverage, while $3 \mathrm{mg} / \mathrm{kg}$ emodin is only sufficient to maintain 1 hour DPP4 inhibition in vivo. In the subsequent experiment, emodin (30 $\mathrm{mg} / \mathrm{kg}$, P.O.) was administered on Balb/C mice $(\mathrm{n}=5)$ or ob/ob (-/-) mice $(\mathrm{n}=5)$. The DPP4 activity, blood glucoselevels and GLP-1 activity were tested 0, 1, 2, 4, 8 hours after administration. The baseline level of DPP4 was higher in ob/ob (-/-) mice compared to Balb/C mice, and the data demonstrated a significant downregulation of DPP4 activity after emodin oral administration in both Balb/C mice and ob/ob (-/-) mice (Fig. 5A). This downregulation maintained 2 hours in Balb/C mice, and 4 hours in ob/ob (-/-) mice, which rebounded afterwards (Fig. 5A). Meanwhile, emodin down-regulated blood glucose level after oral administration. In both Balb/C mice and ob/ob (-/-) mice, emodin treatment significantly downregulated blood glucose levels from baseline (Fig. 5B), and this downregulation maintained for 4 hours post treatment, and the blood glucose levels returned to baseline 8 hours after treatment. Plasma GLP-1 activity was also measured. GLP-1 has a low baseline activity and emodin showed marginal effect on plasma GLP-1 activity (Fig. 5C). These results demonstrate that emodin inhibits DPP4 activity in vivo, which may contribute to its anti-diabetic properties. 


\section{Conclusions}

\section{Supplemental Information}

235 Raw data of screening a natural compound library

236 Raw data of IC50 analysis

237 Raw data of plasma DPP4 activity analysis explain the anti-diabetes effects of this compound. human cancers (National Toxicology 2001). IC50 of $5.7 \mu \mathrm{M}$. the active site of DPP4. mice strains. discovery for the treatment of type 2 diabetes.

Emodin has been detected in various Chinese herbs and is efficacious against inflammatory disorders and cancer (Shrimali et al. 2013; Wei et al. 2013) and liver cirrhosis (Woo et al. 2002); furthermore, emodin has demonstrated immunosuppressive (Kuo et al. 2001) and antibacterial (Wang \& Chung 1997) properties. Although many studies have shown the effects of emodin on metabolic abnormalities (especially diabetes), the molecular mechanisms involved have not been thoroughly studied. Our study shows for the first time that emodin is a selective DPP4 inhibitor both in vitro and in vivo, which may

The toxicity of emodin should also be paid attention to and it has been reported in the previous publications (National Toxicology, 2001; Wang et al. 1997). In these reports, there was no evidence of carcinogenic activity of emodin either in male F344/N rats or female B6C3F1 mice. Although emodin exposure resulted in increased incidences of renal tubule pigmentation in male and female mice and increased incidences of nephropathy in female mice, the emodin doses used in these reports (280 to 2,500 ppm) were much higher than the emodin dose in our reports. Our highest in vivo dose $30 \mathrm{mg} / \mathrm{kg}$ (equivalent to $30 \mathrm{ppm}$ ) is almost one ninth of the lowest dose used in these reports. However, this brings an attention to the chronic toxicity of emodin in the treatment of diabetes in the future. On the other hand, some reports have addressed that emodin isolated from rhubarb may have anti-cancer effects on a few

DPP4 is a well-characterized therapeutic target for type II diabetes treatment, and there have been extensive drug discovery activities reported in this area. However, very few literature has reported natural compounds with activity against DPP4 (Fan et al. 2013). The current study was the first to screen a natural compound library consisting of Chinese herbal medicines and marine organisms, with the goal of identifying novel small molecules that inhibit DPP4. As a result, we discovered that emodin, a compound belonging to the anthraquinone family, selectively inhibited in vitro DPP4 activity with an

To further understand the binding mechanism of emodin and DPP4, we conducted a molecular docking model by simulating the emodin binding mode at the DPP4 active site. The docking assay revealed that emodin interacts with the DPP4 active site and forms H-bonds with Glu205 and Glu206 at

Based on the in vitro data and the docking model, we subsequently conducted animal experiment by orally administering emodin to Balb/C mice and ob/ob (-/-) mice. Plasma DPP4 activity was inhibited by emodin administration in a dose-dependent manner, and the blood glucose levels were decreased in both

Together, these results suggest that emodin is a small molecule inhibitor of DPP4, showing activity both in vitro and in vivo. Emodin, as a novel anti-hypoglycemic compound, may stimulate new drug 


\section{Additional Information and Declarations}

239 Data Availability

240 The following information was supplied regarding data availability:

241 The raw data has been supplied as a Supplemental File.

\section{References}

243 1. Augeri DJ, Robl JA, Betebenner DA, Magnin DR, Khanna A, Robertson JG, Wang A, Simpkins LM, Taunk P, Huang Q, Han SP, Abboa-Offei B, Cap M, Xin L, Tao L, Tozzo E, Welzel GE, Egan DM, Marcinkeviciene J, Chang SY, Biller SA, Kirby MS, Parker RA, and Hamann LG. 2005. Discovery and preclinical profile of Saxagliptin (BMS477118): a highly potent, long-acting, orally active dipeptidyl peptidase IV inhibitor for

2. Baggio LL, and Drucker DJ. 2007. Biology of incretins: GLP-1 and GIP. Gastroenterology 132:2131-2157. 10.1053/j.gastro.2007.03.054

3. Chen T, Zheng LY, Xiao W, Gui D, Wang X, and Wang N. 2015. Emodin ameliorates high glucose induced-podocyte epithelial-mesenchymal transition in-vitro and in-vivo. Cell Physiol Biochem 35:1425-1436. 10.1159/000373963

4. Defronzo RA. 2009. Banting Lecture. From the triumvirate to the ominous octet: a new paradigm for the treatment of type 2 diabetes mellitus. Diabetes 58:773-795. 10.2337/db09-9028

5. Eckhardt M, Langkopf E, Mark M, Tadayyon M, Thomas L, Nar H, Pfrengle W, Guth B, Lotz R, Sieger P, Fuchs H, and Himmelsbach F. 2007. 8-(3-(R)-aminopiperidin-1-yl)-7but-2-ynyl-3-methyl-1-(4-methyl-quinazolin-2-ylme thyl)-3,7-dihydropurine-2,6-dione (BI 1356), a highly potent, selective, long-acting, and orally bioavailable DPP-4 inhibitor

6. Fan J, Johnson MH, Lila MA, Yousef and Mejia EG.2013. Berry and Citrus Phenolic Compounds Inhibit Dipeptidyl Peptidase IV: Implications in Diabetes Management. Evid Based Complement Alternat Med 2013:479505. 10.1155/2013/479505

7. Feng J, Zhang Z, Wallace MB, Stafford JA, Kaldor SW, Kassel DB, Navre M, Shi L, Skene RJ, Asakawa T, Takeuchi K, Xu R, Webb DR, and Gwaltney SL, 2nd. 2007. Discovery of alogliptin: a potent, selective, bioavailable, and efficacious inhibitor of dipeptidyl peptidase IV. J Med Chem 50:2297-2300. 10.1021/jm0701041

271

272

273 Emodin, a natural product, selectively inhibits 11 beta-hydroxysteroid dehydrogenase type 1 and ameliorates metabolic disorder in diet-induced obese mice. Br J Pharmacol 161:113-126. 10.1111/j.1476-5381.2010.00826.x

9. Geng Y, Lu ZM, Huang W, Xu HY, Shi JS, and Xu ZH. 2013. Bioassay-guided isolation of DPP-4 inhibitory fractions from extracts of submerged cultured of Inonotus obliquus. Molecules 18:1150-1161. 10.3390/molecules18011150

10. Hopsu-Havu VK, and Glenner GG. 1966. A new dipeptide naphthylamidase hydrolyzing glycyl-prolyl-beta-naphthylamide. Histochemie 7:197-201.

11. Huang WJ, Niu HS, Lin MH, Cheng JT, and Hsu FL. 2010. Antihyperglycemic effect of catalpol in streptozotocin-induced diabetic rats. J Nat Prod 73:1170-1172.

280 $10.1021 / \mathrm{np} 9008317$ 
281 12. Ji X, Xia C, Wang J, Su M, Zhang L, Dong T, Li Z, Wan X, Li J, Li J, Zhao L, Gao Z,

282

283

284

285

286

287

288

289

290

291

292

293

294

295

296

297

298

299

300

301

302

303

304

305

306

307

308

309

310

311

312

313

314

315

316

317

318

319

320

321

322

323

324

325

326

Jiang H, and Liu H. 2014. Design, synthesis and biological evaluation of 4fluoropyrrolidine-2-carbonitrile and octahydrocyclopenta[b]pyrrole-2-carbonitrile derivatives as dipeptidyl peptidase IV inhibitors. Eur J Med Chem 86:242-256. 10.1016/j.ejmech.2014.08.059

13. Kim D, Wang L, Beconi M, Eiermann GJ, Fisher MH, He H, Hickey GJ, Kowalchick JE, Leiting B, Lyons K, Marsilio F, McCann ME, Patel RA, Petrov A, Scapin G, Patel SB, Roy RS, Wu JK, Wyvratt MJ, Zhang BB, Zhu L, Thornberry NA, and Weber AE. 2005. (2R)-4-oxo-4-[3-(trifluoromethyl)-5,6-dihydro[1,2,4]triazolo[4,3-a]pyrazin-7(8H)- yl]-1(2,4,5-trifluorophenyl)butan-2-amine: a potent, orally active dipeptidyl peptidase IV inhibitor for the treatment of type 2 diabetes. J Med Chem 48:141-151. 10.1021/jm0493156

14. Kuo YC, Tsai WJ, Meng HC, Chen WP, Yang LY, and Lin CY. 2001. Immune reponses in human mesangial cells regulated by emodin from Polygonum hypoleucum Ohwi. Life Sci 68:1271-1286.

15. Lambeir AM, Durinx C, Scharpe S, and De Meester I. 2003. Dipeptidyl-peptidase IV from bench to bedside: an update on structural properties, functions, and clinical aspects of the enzyme DPP IV. Crit Rev Clin Lab Sci 40:209-294. 10.1080/713609354

16. Lankas GR, Leiting B, Roy RS, Eiermann GJ, Beconi MG, Biftu T, Chan CC, Edmondson S, Feeney WP, He H, Ippolito DE, Kim D, Lyons KA, Ok HO, Patel RA, Petrov AN, Pryor KA, Qian X, Reigle L, Woods A, Wu JK, Zaller D, Zhang X, Zhu L, Weber AE, and Thornberry NA. 2005. Dipeptidyl peptidase IV inhibition for the treatment of type 2 diabetes: potential importance of selectivity over dipeptidyl peptidases 8 and 9. Diabetes 54:2988-2994.

17. Lin YJ, Hu G, Li KJ, Zhao YF, Wei J, and Zhen YZ. 2015. The protection of Rhein lysinate to liver in diabetic mice induced by high-fat diet and streptozotocin. Arch Pharm Res 38:885-892. 10.1007/s12272-014-0423-4

18. Liu JP, Zhang M, Wang WY, and Grimsgaard S. 2004. Chinese herbal medicines for type 2 diabetes mellitus. Cochrane Database Syst Rev:CD003642.

10.1002/14651858.CD003642.pub2

19. Mulvihill EE, and Drucker DJ. 2014. Pharmacology, physiology, and mechanisms of action of dipeptidyl peptidase-4 inhibitors. Endocr Rev 35:992-1019. 10.1210/er.20141035

20. National Toxicology P. 2001. NTP Toxicology and Carcinogenesis Studies of EMODIN (CAS NO. 518-82-1) Feed Studies in F344/N Rats and B6C3F1 Mice. Natl Toxicol Program Tech Rep Ser 493:1-278.

21. Ramos-Zavala MG, Gonzalez-Ortiz M, Martinez-Abundis E, Robles-Cervantes JA, Gonzalez-Lopez R, and Santiago-Hernandez NJ. 2011. Effect of diacerein on insulin secretion and metabolic control in drug-naive patients with type 2 diabetes: a randomized clinical trial. Diabetes Care 34:1591-1594. 10.2337/dc11-0357

22. Shrimali D, Shanmugam MK, Kumar AP, Zhang J, Tan BK, Ahn KS, and Sethi G. 2013. Targeted abrogation of diverse signal transduction cascades by emodin for the treatment of inflammatory disorders and cancer. Cancer Lett 341:139-149. 10.1016/j.canlet.2013.08.023

23. Smith EP, An Z, Wagner C, Lewis AG, Cohen EB, Li B, Mahbod P, Sandoval D, PerezTilve D, Tamarina N, Philipson LH, Stoffers DA, Seeley RJ, and D'Alessio DA. 2014. 
327

328

329

330

331

332

333

334

335

336

337

338

339

340

341

342

343

344

345

346

347

348

349

350

351

352

353

354

355

356

357

358

359

360

361

362

363

364

365

366

367

368

369

370

The role of beta cell glucagon-like peptide-1 signaling in glucose regulation and response to diabetes drugs. Cell Metab 19:1050-1057. 10.1016/j.cmet.2014.04.005

24. Song P, Kim JH, Ghim J, Yoon JH, Lee A, Kwon Y, Hyun H, Moon HY, Choi HS, Berggren PO, Suh PG, and Ryu SH. 2013. Emodin regulates glucose utilization by activating AMP-activated protein kinase. J Biol Chem 288:5732-5742. 10.1074/jbc.M112.441477

25. Villhauer EB, Brinkman JA, Naderi GB, Burkey BF, Dunning BE, Prasad K, Mangold BL, Russell ME, and Hughes TE. 2003. 1-[[(3-hydroxy-1-adamantyl)amino]acetyl]-2cyano-(S)-pyrrolidine: a potent, selective, and orally bioavailable dipeptidyl peptidase IV inhibitor with antihyperglycemic properties. J Med Chem 46:2774-2789.

$10.1021 / \mathrm{jm} 0300911$

26. Wang HH, and Chung JG. 1997. Emodin-induced inhibition of growth and DNA damage in the Helicobacter pylori. Curr Microbiol 35:262-266.

27. Wang HJ, and Chiang BH. 2012. Anti-diabetic effect of a traditional Chinese medicine formula. Food Funct 3:1161-1169. 10.1039/c2fo30139c

28. Wang N, Zhang D, Mao X, Zou F, Jin H, and Ouyang J. 2009. Astragalus polysaccharides decreased the expression of PTP1B through relieving ER stress induced activation of ATF6 in a rat model of type 2 diabetes. Mol Cell Endocrinol 307:89-98. 10.1016/j.mce.2009.03.001

29. Wang YJ, Huang SL, Feng Y, Ning MM, and Leng Y. 2012. Emodin, an 11 betahydroxysteroid dehydrogenase type 1 inhibitor, regulates adipocyte function in vitro and exerts anti-diabetic effect in ob/ob mice. Acta Pharmacol Sin 33:1195-1203. 10.1038/aps.2012.87

30. Wei WT, Lin SZ, Liu DL, and Wang ZH. 2013. The distinct mechanisms of the antitumor activity of emodin in different types of cancer (Review). Oncol Rep 30:25552562. 10.3892/or.2013.2741

31. Woo SW, Nan JX, Lee SH, Park EJ, Zhao YZ, and Sohn DH. 2002. Aloe emodin suppresses myofibroblastic differentiation of rat hepatic stellate cells in primary culture. Pharmacol Toxicol 90:193-198.

32. Wu Z, Chen Q, Ke D, Li G, and Deng W. 2014. Emodin protects against diabetic cardiomyopathy by regulating the AKT/GSK-3beta signaling pathway in the rat model. Molecules 19:14782-14793. 10.3390/molecules190914782

33. Xie W, and Du L. 2011. Diabetes is an inflammatory disease: evidence from traditional Chinese medicines. Diabetes Obes Metab 13:289-301. 10.1111/j.14631326.2010.01336.x

34. Xue J, Ding W, and Liu Y. 2010. Anti-diabetic effects of emodin involved in the activation of PPARgamma on high-fat diet-fed and low dose of streptozotocin-induced diabetic mice. Fitoterapia 81:173-177. 10.1016/j.fitote.2009.08.020

35. Zhao XY, Qiao GF, Li BX, Chai LM, Li Z, Lu YJ, and Yang BF. 2009. Hypoglycaemic and hypolipidaemic effects of emodin and its effect on L-type calcium channels in dyslipidaemic-diabetic rats. Clin Exp Pharmacol Physiol 36:29-34. 10.1111/j.14401681.2008.05051.x 
371

372

373

374

375

376

377

378

379

380

381

382

383

384

385 
Figure 1

Emodin was found to inhibit DPP4 activity after screening a natural compound library.
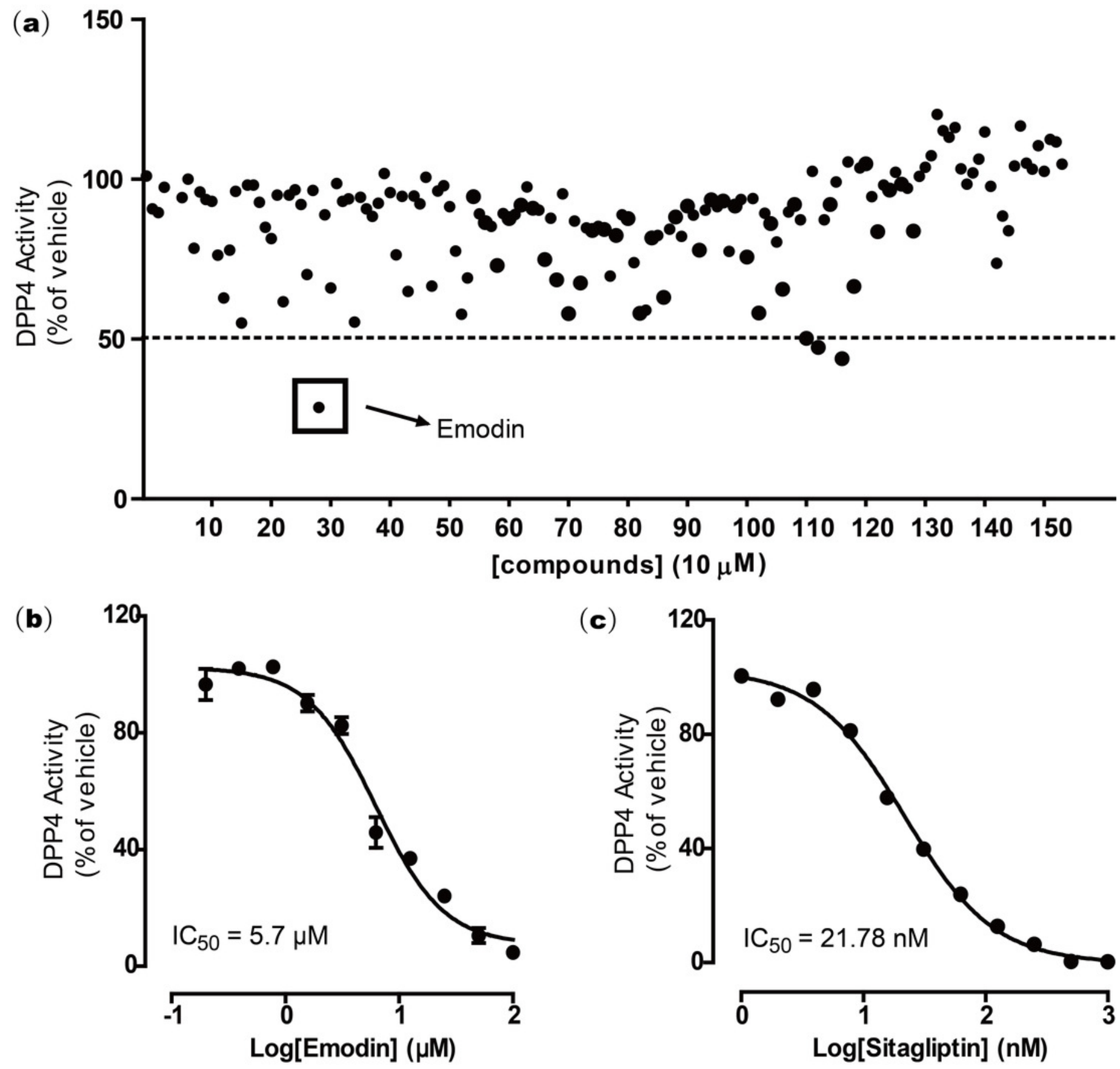
Figure 2

Anthraquinone compounds do not inhibit either (a) DPP8 or (b) DPP9.
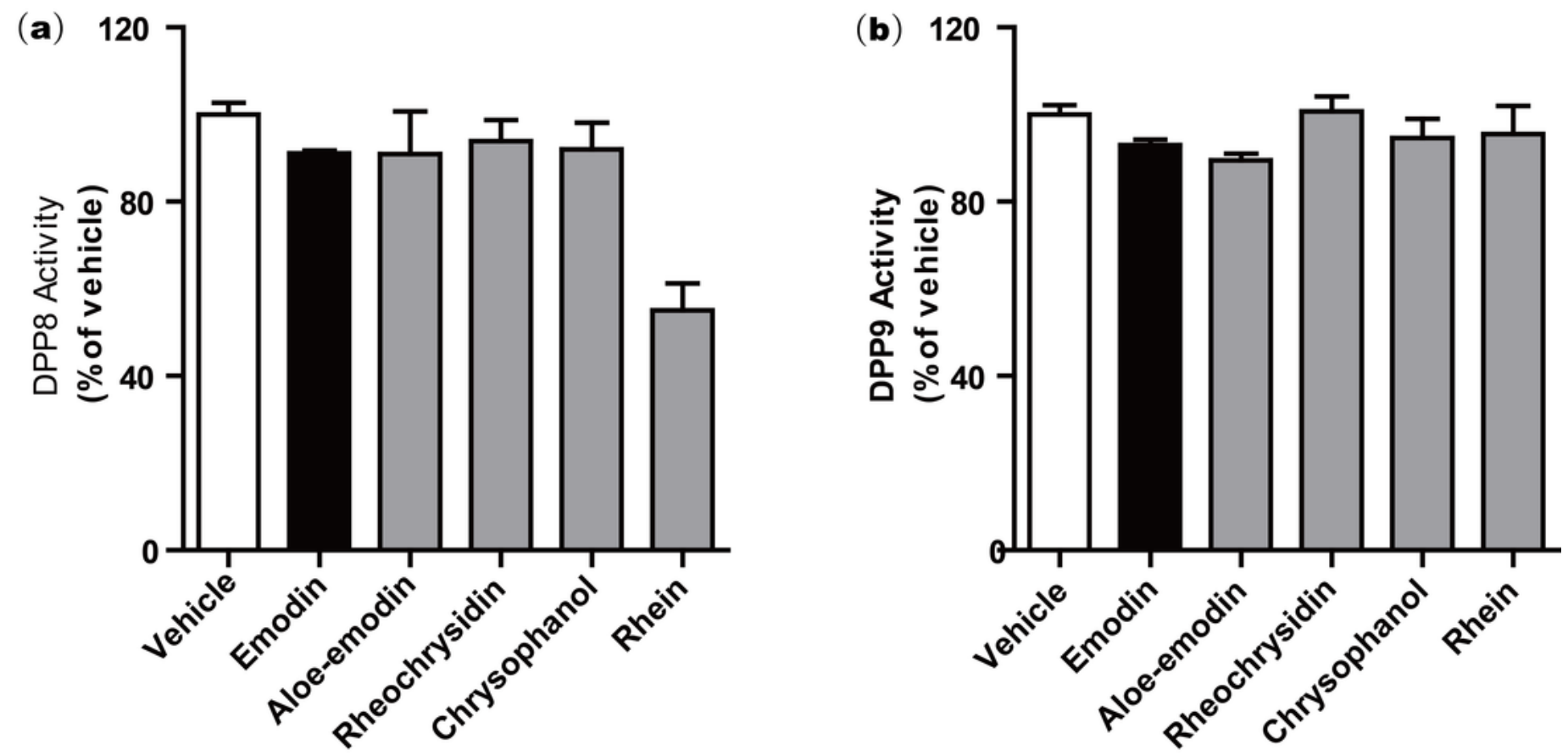
Figure 3

Docking model reveals the binding mode of emodin to DPP4 protein.

(a)

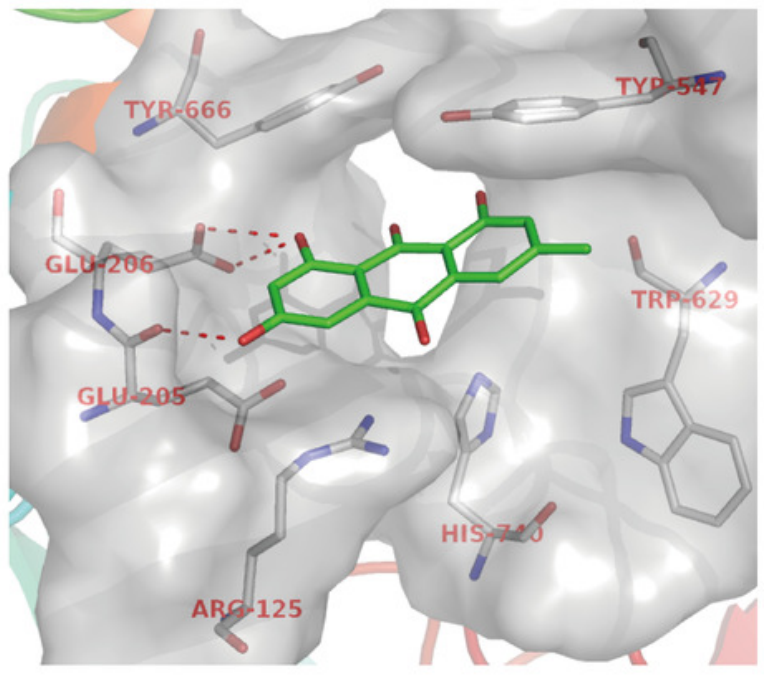

(b)

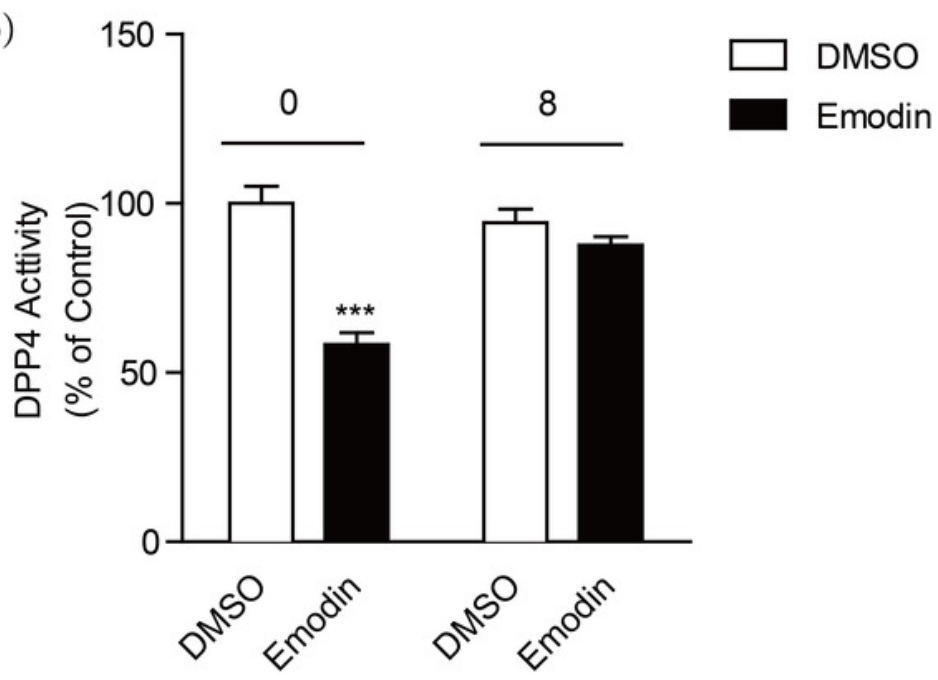


Figure 4

Emodin treatment $(3,10$ and $30 \mathrm{mg} / \mathrm{kg}$, P.O.) in mice decreased the plasma DPP4 activity in a dose-dependent manner.

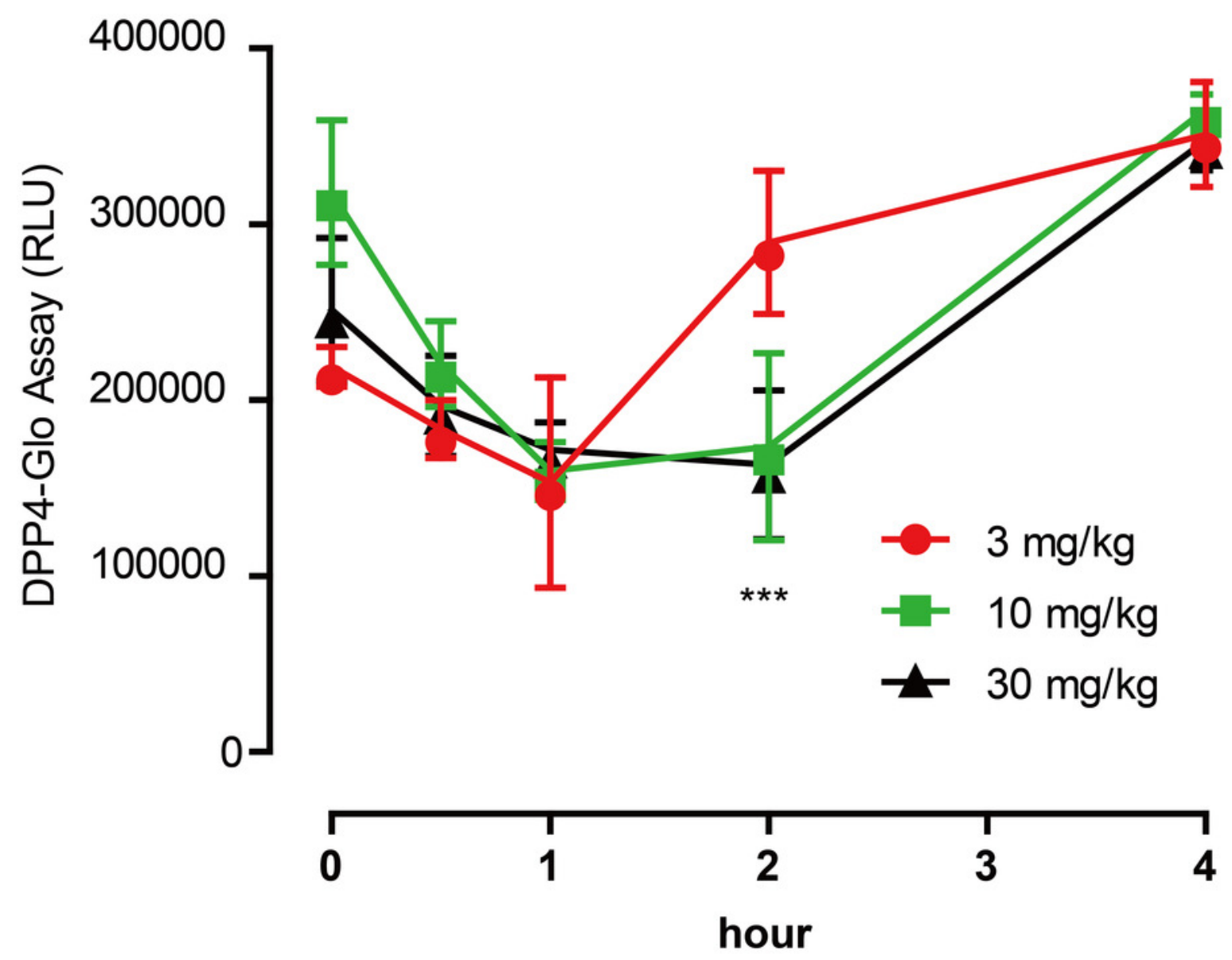


Figure 5

Emodin treatment in mice.
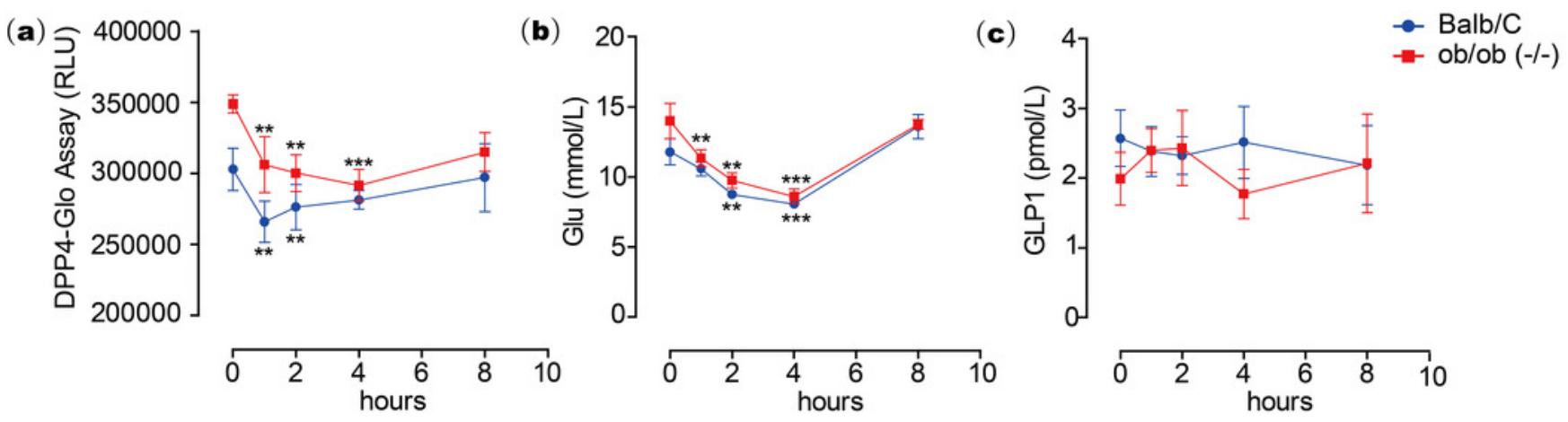


\section{Table 1 (on next page)}

Anthraquinone compounds inhibit DPP4 activity. 
1

2

\begin{tabular}{cccccc}
\hline Compound & $\mathbf{R}_{\mathbf{1}}$ & $\mathbf{R}_{\mathbf{2}}$ & $\begin{array}{c}\mathbf{I C}_{50} \text { of DPP4 } \\
\text { Inhibition }(\mu \mathrm{M})\end{array}$ & $\mathbf{K i}(\mu \mathbf{M})$ & $\begin{array}{c}\text { Binding Energy } \\
(\mathbf{k c a l} / \mathbf{m o l})\end{array}$ \\
\hline Emodin & $-\mathrm{CH}_{3}$ & $-\mathrm{OH}$ & $5.76 \pm 0.42$ & $0.85 \pm 0.06$ & -5.19 \\
Aloe-emodin & $-\mathrm{H}$ & - & $16.02 \pm 4.24$ & $2.37 \pm 0.62$ & -5.31 \\
Rheochrysidin & $-\mathrm{CH}_{3}$ & $-\mathrm{OCH}_{3} \mathrm{OH}$ & $>100$ & - & -4.60 \\
Chrysophanol & $-\mathrm{H}$ & $-\mathrm{CH}_{3}$ & $>100$ & - & -4.46 \\
& & - & & & \\
Rhein & $-\mathrm{H}$ & $\mathrm{O}$ & $23.06 \pm 3.57$ & $3.42 \pm 0.53$ & -4.73 \\
& & $\mathrm{O}$ & & \\
\hline
\end{tabular}

4

5

6

7

8

9

10

11

12

13

14

Table 1

Anthraquinone compounds inhibit DPP4 activity.

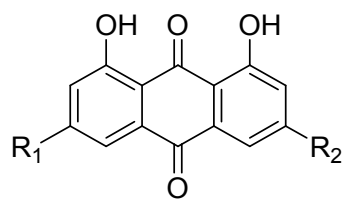

Values $(\mu \mathrm{M})$ are means \pm SE.

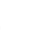

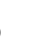

8

9

(1) 
